\title{
Social Information Processing Skills in Children with Histories of Heavy Prenatal Alcohol Exposure
}

\author{
Christie L. McGee • Olivia A. Bjorkquist • \\ Joseph M. Price • Sarah N. Mattson • Edward P. Riley
}

Published online: 13 March 2009

(C) The Author(s) 2009. This article is published with open access at Springerlink.com

\begin{abstract}
Based on caregiver report, children with prenatal alcohol exposure have difficulty with social functioning, but little is known about their social cognition. The current study assessed the social information processing patterns of school-age children with heavy prenatal alcohol exposure using a paradigm based on Crick and Dodge's reformulated six-stage model. Fifty-two children (aged 7-11) with and without heavy prenatal alcohol exposure were tested using a structured interview measure of social information processing involving 18 videotaped vignettes of children in group entry and provocation situations. Alcohol-exposed children displayed maladaptive processing patterns on the goal, response generation, and response evaluation steps in group entry situations, and encoding, attribution, response evaluation, and enactment steps during provocation situations. Children with heavy prenatal alcohol exposure also had difficulty on the Test of Problem Solving, and performance correlated with social information processing measures. Such difficulties may lead to problems in social functioning and warrant early intervention.
\end{abstract}

C. L. McGee $(\bowtie) \cdot$ O. A. Bjorkquist $\cdot$ S. N. Mattson $\cdot$ E. P. Riley Center for Behavioral Teratology, Department of Psychology,

San Diego State University,

6363 Alvarado Ct. Suite 200,

San Diego, CA 92120, USA

e-mail: cmcgee@projects.sdsu.edu

C. L. McGee

SDSU/University of California-San Diego Joint

Doctoral Program in Clinical Psychology,

San Diego, CA, USA

J. M. Price

Department of Psychology, San Diego State University,

San Diego, CA 92120, USA
Keywords Fetal alcohol syndrome $\cdot$ Prenatal alcohol exposure $\cdot$ Social information processing $\cdot$ Social functioning

\section{Introduction}

Prenatal exposure to alcohol is a major public health concern and can lead to life-long impairments in cognition and behavior. One outcome of heavy prenatal alcohol exposure is the fetal alcohol syndrome (FAS), characterized by dysmorphic facial features, growth deficiency, and central nervous system dysfunction (Hoyme et al. 2005; Jones and Smith 1973). FAS is not the only consequence of prenatal alcohol exposure; the term fetal alcohol spectrum disorders (FASD) encompasses the full range of effects, and is estimated to occur in $1 \%$ of all live births (Sampson et al. 1997). Heavily exposed children with and without FAS often show similar cognitive and behavioral performance (e.g., Mattson et al. 1998) and may have impairments in multiple cognitive domains including intelligence, attention, language, visual-spatial skills, learning and memory, motor functioning, and executive skills (Kodituwakku 2007).

One area not widely studied in this population is social problem solving skills. Retrospectively-identified children with prenatal alcohol exposure demonstrate significant impairments in social functioning as rated by their caregivers (Thomas et al. 1998; Whaley et al. 2001). In addition, studies consistently find deficits in socialization above and beyond intellectual impairment (Thomas et al. 1998) or the presence of problems requiring clinical intervention (Whaley et al. 2001). On self-report indices, alcohol-exposed adolescents endorse difficulties with social problem solving, which include approaching problems with a pessimistic orientation, a low frustration tolerance, and a tendency to utilize an avoidant, careless, or impulsive 
approach to solving their everyday problems (McGee et al. 2008). The only systematic observational study of this population found that alcohol-exposed children display a normal frequency of social interactions with appropriate facial expressions and nonverbal behaviors, but the quality of their social interactions is often poor and inappropriate (Bishop et al. 2007). To date, no studies have directly assessed the social information processing skills of children with prenatal alcohol exposure.

\section{Social Information Processing}

During childhood, common types of social challenges include initiating friendships, acquiring objects, seeking and offering help, seeking attention or information, and stopping others from acting in an undesirable way (Rubin and Krasnor 1986). There is a growing body of empirical evidence linking children's social behavior with the manner in which they process social information (Crick and Dodge 1994). One of the theoretical models guiding this line of research was developed by Dodge (1980) and later elaborated by Crick and Dodge (1994). This nonlinear, reciprocal model (see Fig. 1; Crick and Dodge 1994) involves six steps. During Steps 1 and 2, children selectively attend to particular situational and internal cues, encode those cues, and interpret them utilizing processes such as accessing a personalized mental representation of situational cues stored in long term memory (Crick and Dodge 1994). In Step 3, children select goals or desired

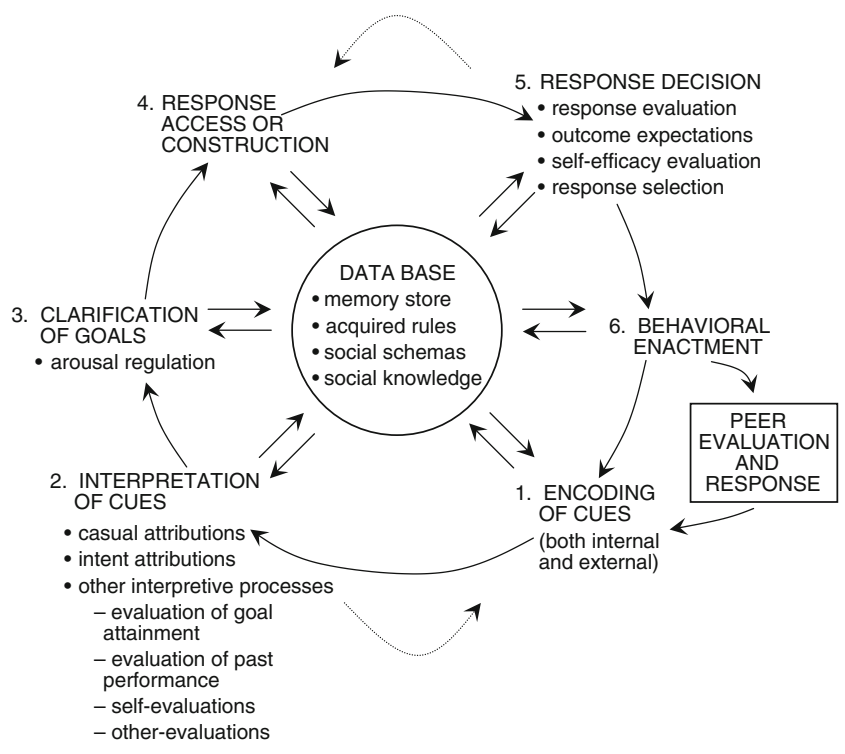

Fig. 1 A social information processing model of children's social adjustment. Note: From "A review and reformulation of socialinformation-processing mechanisms of children's social adjustment," by N. R. Crick \& K. A. Dodge (1994), Psychological Bulletin, 115, p.74. Copyright 1994 by the American Psychological Association. Reprinted with permission outcomes for the situation, and then in Step 4, children either access possible responses from memory or, if a situation is novel, construct new behaviors in response to immediate social cues. Children then evaluate the potential outcomes of these responses and their ability to carry them out in Step 5 and select the most positively evaluated response, which is enacted in Step 6. While this model may lead to the view of children as conscious, reflective processors, the majority of processing is likely highly automated.

According to this model, children approach a social situation with a set of biologically limited capabilities and memories of previous experiences (illustrated by the "database" in the center of the model; Crick and Dodge 1994), and the relationship between these capabilities and information represented by the database and the social information processing steps is reciprocal. There is evidence indicating that social information processing and problem solving are situation-specific (Dodge et al. and Conduct Problems Prevention Research Group 2002). Knowledge structures within the "database" guide behavior and facilitate the representation of the current event by filling in missing information from existing memory stores and supplying information regarding past consequences for similar situations (Burks et al. 1999). Thus, children who have limited experiences in social situations, often due to isolation or rejection, tend to have less varied knowledge structures to draw upon and less practice using these structures when processing information in social interactions.

According to the revised Crick and Dodge model social information processing proceeds in simultaneous parallel paths such that children are concurrently encoding, interpreting, and accessing responses. However, the path for a specific stimulus to a behavioral response follows a timerelated linear sequence of steps. Thus, deficits in one step of processing can affect adequate functioning in other steps. For example, poor encoding may result in biased interpretations of the peer's intent if the child did not encode the appropriate social cues. To adequately examine social information processing patterns, a comprehensive assessment of the six social information processing steps is essential (Crick and Dodge 1994).

The goal of this study was to investigate the social information processing patterns of children with heavy prenatal exposure to alcohol, with and without FAS, utilizing the Crick and Dodge (1994) social information processing model as a guide. This study focused on two areas of interpersonal problem solving common to children: peer group entry and provocation by a peer. Children also completed the Test of Problem Solving, Third Edition (TOPS-3), to provide additional information on social problem solving and assess concurrent validity. It was hypothesized that alcohol-exposed children would display significant difficulties in both domains of social informa- 
tion processing and the TOPS-3, and that performance on the TOPS-3 would be related to social information processing indices.

\section{Methods}

Participants

Two groups of children (aged 7-11) were included in this study: children with heavy prenatal alcohol exposure (ALC, $n=26)$ and a typically developing control group (CON, $n=$ 26). Groups were matched on age, sex, socioeconomic status (SES), and race/ethnicity (See Table 1). Children were recruited as part of a larger ongoing study of the behavioral teratogenicity of alcohol. Alcohol-exposed children are recruited into this larger study via several mechanisms, including professional referral or selfreferral. Non-exposed participants are recruited from the community via advertising at various agencies and childrelated venues (e.g., health fairs, child care centers), as well as referrals by other participating families. Teratogenic exposure history was determined through multi-source collateral report, including review of available medical, social service, and adoption agency records and maternal report, when available. Heavy exposure was defined as at least 4 drinks per occasion at least once per week or 14 drinks per week during pregnancy. Children in the alcoholexposed group were also evaluated by a dysmorphologist with expertise in alcohol teratogenesis. Alcohol-exposed children with $(n=6)$ or without $(n=18)$ a diagnosis of FAS were included in the ALC group. Two children had heavy

Table 1 Demographic Information for Non-exposed Control (CON) and Alcohol-exposed (ALC) Groups

\begin{tabular}{lcc}
\hline & CON & \multicolumn{2}{c}{ ALC } \\
\hline $\mathrm{N}$ & 26 & 26 \\
\# FAS & $\mathrm{n} / \mathrm{a}$ & $6^{\mathrm{a}}$ \\
Sex [N (\% Female)] & $13(50.0)$ & $12(46.2)$ \\
Race [N (\% White)] & $19(73.1)$ & $16(61.5)$ \\
Ethnicity [N (\% Hispanic)] & $7(26.9)$ & $6(23.1)$ \\
Home [N (\% Biological)] & $25(96.2)$ & $2(7.7)$ \\
[N (\% Adoptive)] & $1(3.8)$ & $18(69.2)$ \\
[N (\% Relative)] & $0(0.0)$ & $4(15.4)$ \\
[N (\% Foster)] & $0(0.0)$ & $2(7.7)$ \\
Age in Years [Mean (SD)] & $10.03(1.20)$ & $9.42(1.58)$ \\
SES [Mean (SD)] & $47.58(11.39)$ & $43.73(15.47)$ \\
FSIQ [Mean (SD)] & $107.83(13.05)$ & $91.29(18.02)$ \\
\hline
\end{tabular}

${ }^{a}$ Two children in the ALC group had not been seen by a dysmorphologist at study completion; ${ }^{\mathrm{b}}$ Five children were missing FSIQ data $(3 \mathrm{CON}, 2$ ALC) exposure but had not been evaluated by a dysmorphologist upon study completion. Exclusionary criteria for both groups were primary language other than English, head injury with loss of consciousness greater than $30 \mathrm{~min}$, and other disabling psychiatric or physical disorders that would prohibit participation (e.g., psychoses, paralysis).

\section{Procedure}

Testing was completed at the Center for Behavioral Teratology (CBT) or at or near the child's home if travel to the CBT posed a significant burden to the family. Children completed the Social Information Processing Interview and the TOPS-3. In addition, IQ scores from the WISC-III were available for most children from other studies. Socioeconomic status (SES) was measured by the Hollingshead Four Factor Index of Social Status (Hollingshead 1975). Informed consent was obtained from the parent or guardian and assent from the child, and children were provided with an incentive (money or a toy of similar value) for their participation. All procedures were approved by the Institutional Review Boards at San Diego State University and University of California, San Diego.

\section{Measures}

\section{Social Information Processing Interview}

Based on measures created by Dodge (Dodge 1980; Dodge et al. 2002), the social information processing interview used in this investigation was developed by Price and colleagues (Keil and Price 2009) to reflect the broader range of ethnic groups found in San Diego County. With this measure, each child was presented with 18 videotaped vignettes of problematic social situations, and asked to imagine that they are the focal character. Child actors from four major ethnic groups (Caucasian, Latino, AfricanAmerican, and Asian) were included, with ethnicity being randomized across stories. Videos included male or female actors and sex was equally represented across the 18 scenarios. All participants in the study viewed the same videos. Two domains of problematic social situations were presented: peer group entry and response to provocation. Each domain was composed of nine stories. In the hypothetical stories depicting Provocation situations, the focal child experiences a negative outcome in a social situation (e.g., hit by a ball). In Group Entry situations, the focal child approaches two peers involved in a social activity. The intent of the other child(ren) varied across stories and was either benign, ambiguous, or hostile (3 stories of each intent per domain). Following each video clip, the child completed a standardized interview that involved both forced choice and free response options 
corresponding to the six-step model of social information processing presented above. Further coding details and examples of responses can be found in the Appendix.

Step 1: Encoding Children were asked, "What happened in the story?" Responses were coded according to the number of details provided. Scores from the stories were summed and averaged, within social domains, to arrive at a single encoding score for each of the two domains.

Step 2: Interpretation In the Provocation domain, children were asked why the other child acted the way that $\mathrm{s} /$ he did in order to assess their attribution of peer intent. Children were asked to choose from the following intent options: (1) being mean, (2) not being mean, (3) hard to tell, or (4) don't know. Hostile attribution was calculated as the proportion of times that a child responded, "being mean" in either benign or ambiguous stories. Non-hostile attribution was calculated as the proportion of times that a child responded, "not being mean" in either hostile or ambiguous stories. Responses of "hard to tell" were not included in this calculation to create a variable reflecting a level of certainty in children's non-hostile attributions. In the Group Entry domain, children were asked how much the other kids want to play with them: (1) not at all, (2) just a little, or (3) very much. Hostile attribution was calculated as the proportion of times that a child responded, "not at all" in either benign or ambiguous stories. Nonhostile attribution was calculated as the proportion of times that a child responded, "just a little" or "very much" in either hostile or ambiguous stories.

Step 3: Clarification or Selection of a Goal Children were asked, "What do you want to happen next?" One only goal was solicited from children. For both domains, responses were coded as pro-social, non-social, negative/aggressive, inept, or irrelevant. The proportion of each response across stories was calculated for each domain.

Step 4: Response Generation To control for differences in interpretation of intent, at this point in the interview the interviewer told children the intent of the peer(s). Children's problem-solving patterns were then assessed by asking, "What would you say or do if this happened to you?" until either six responses were obtained or the child had no additional responses. Responses were then coded as aggressive (e.g., hit the peer), competent (e.g., ask the peer why they did that), or inept (e.g., pout or whine). The aggressive problem-solving variable was created as the proportion of aggressive responses over the total number of responses made in each domain. The competent and inept problem-solving variables were created in the same manner. Similar proportions counting only non-repeated responses were also formed. Finally the quality of the first response given was assessed as the number of aggressive, competent, or inept first responses divided by the total number of stories per domain.

Step 5: Response Evaluation Children viewed three possible response strategies (i.e., aggressive, competent, and inept) and were asked specific questions to assess if each response would be effective in obtaining both affiliation (i.e., whether the other child(ren) would like them if they performed that specific behavior) and instrumental (i.e., whether the behavior would be effective in obtaining the desired outcome) outcomes. After responding "yes" or "no," children stated if they were "a little sure" or "really sure" of their response, resulting in four possible response options. Evaluation variables were proportions of each participant's endorsement of a strategy type (either aggressive, competent, or inept) to the total endorsement score, separately for affiliation or instrumental outcomes.

Step 6: Enactment For each vignette, the subject was asked to role-play a competent response to the event. Responses were coded on a scale of 0 to 4 , based on appropriateness/ relevancy of response, eye contact, and tone of voice. Scores from the stories within each domain were summed and averaged to arrive at a single enactment score for each of the two domains.

Reliability Inter-rater agreement was calculated for steps requiring subjective ratings or response coding. Ten files were selected at random and percent agreement (based on initial ratings) was determined for each story (see Table 2). Values were similar to or higher than previous studies (e.g., Dodge et al. 2003; Price and Landsverk 1998; Quiggle et al. 1992). A previous study estimated median alpha values for this measure at .74 and .79 for Provocation and Group Entry
Table 2 Percentage Agreement Between Independent Raters on Social Information Processing Steps

\begin{tabular}{lcccc}
\hline Step & Group Entry & Provocation & Mean & Range \\
\hline Encoding & 88.9 & 83.3 & 86.1 & $70-100$ \\
Goal Clarification & 100.0 & 98.9 & 99.4 & $90-100$ \\
Response Generation (individual codes) & 85.1 & 87.5 & 86.3 & $73-98$ \\
Response Generation (overall category) & 93.8 & 98.7 & 96.2 & $82-100$ \\
Enactment & 91.1 & 85.6 & 88.3 & $70-100$ \\
\hline
\end{tabular}


variables, respectively, supporting the generally strong reliability of the processing variables (Keil and Price 2009).

Test of Problem Solving, Third Edition Elementary Version (TOPS-3)

The TOPS-3 (Bowers et al. 2005) measures a child's languagebased problem solving and critical thinking skills and contains six scales including Making Inferences, Predicting, Determining Causes, Sequencing, Negative Questions, and Problem Solving, as well as a Total summary score. During the test, the child is presented with 18 photographs of various situations (e.g., child sick laying in bed, basketball practice, firefighter and small child) and is asked specific questions corresponding to the six scales. Children's responses received 2, 1 , or 0 points, depending on the appropriateness of the content, semantics, and linguistics. Internal consistency using KuderRichardson coefficients and averages across age groups range from .56 for Predicting to .69 for Negative Questions and Sequencing (Bowers et al. 2005).

\section{Statistical Analyses}

Prior to analyses, data were examined for the presence of missing data, outliers, and the assumptions for analysis of variance. No systematic outliers were identified on the social information processing measure and all data points were retained. Multiple social information processing variables exhibited marked deviations for normality, especially for goal and response generation steps. For the most part, the shape of the distributions did not differ across groups. Multiple transformations were applied to variables (e.g., arcsine, square root), but did not substantially improve normality or affect results of significance testing. Results of nonparametric tests (e.g., Mann-Whitney) were compared to standard analysis of variance (ANOVA) results and did not markedly differ. The assumption of homogeneity of variance was not markedly violated. Group differences for demographic variables were examined through Chi-Square (sex, race, ethnicity, living environment) and univariate ANOVA (age, SES, FSIQ) techniques.

Due to the large number of social information processing variables, data reduction techniques were attempted through principal components analyses. However, due to variables loading on multiple components and low interpretability of individual components, this approach was discontinued. Examination of zero-order correlations revealed that correlations varied considerably in magnitude within steps and therefore a theoretical approach utilizing multivariate ANOVA to simultaneously examine variables within steps would not be incrementally beneficial. For this reason, a univariate approach to ANOVA was selected. Due to the increased probability of making a type one error with multiple univariate comparisons, group differences were only considered if they represented medium to large effects according to Cohen's criteria ( $>0.5$; Cohen 1988). To explore the effects of age and sex on social information processing, zero-order correlations were examined. When significantly correlated, age or sex was included in univariate ANOVA models as either a covariate or explanatory variable, respectively.

Group differences on TOPS-3 scales were examined using the same methods as discussed for the social information processing variables. In addition, the relationships between problem solving variables on the TOPS-3 and social information processing measure were examined through canonical correlation, separately for Group Entry and Provocation domains. Social information processing variables were selected for inclusion in specific models if their zeroorder correlations were between .3 and .7 for variables representing the given construct and correlations within groups were not markedly different. Canonical correlation values $\left(r_{c}\right)$ and fit statistics are provided in Tables 6 and 7 and only canonical pairs accounting for a significant amount of variance are discussed in the text. A measure of effect size $\left(\eta^{2}\right)$ is provided. Interpretation of significant canonical variate pairs was determined through examination of standardized canonical correlation coefficients and canonical loadings for each set. A cutoff value of greater than |.30| was utilized for practical significance for both indices.

Two supplemental analyses were also completed. Children with prenatal alcohol exposure often have lower IQ scores than typically developing children and this was true in the current sample. We examined the influence of IQ on social information processing skills by individually matching 10 children from each group on age, sex, and FSIQ [Means: ALC 102.90, CON 102.60]. Because of small sample sizes and the preliminary nature of the analyses, only descriptive data and effect sizes are presented. Second, the performance of alcoholexposed children with and without FAS was compared to determine if diagnosis accounted for some of the variability in social information processing scores. The two children in the ALC group who had not been seen by a dysmorphologist were excluded from these analyses.

\section{Results}

Group Differences on Demographic Variables

Groups did not significantly differ with respect to sex, race, ethnicity, age, or SES ( $p$ 's $>0.05)$. Groups differed significantly on living environment $\left(\chi^{2}(1)=40.75, p<0.001\right)$, as only 2 children in the ALC group lived with a biological parent. Groups also differed significantly on FSIQ $(F(1,46)=12.89$, 
$p=0.001$ ), with children in the ALC group having lower FSIQ scores on average than children in the CON group. These findings are consistent with previous studies.

\section{Group Differences on Social Information Processing}

Group Entry Group differences were observed in the goal selection, response generation, and response evaluation steps of social information processing (see Table 3).
Specifically, the ALC group gave fewer pro-social goals and more inept goals than the CON group, though the difference on the proportion of inept goals was due to responses from five children in the ALC group; no child in the CON group provided any inept goals. During the response generation step, groups did not differ with respect to total number of responses given or overall quality of these responses. However, alcohol-exposed children generated a higher proportion of aggressive responses and a
Table 3 Means, Standard Deviations, Effect Sizes, and Significance Testing Results for Non-exposed Controls (CON) and Children with Prenatal Alcohol Exposure (ALC) on Social Information Processing Variables in Group Entry Situations

\begin{tabular}{|c|c|c|c|c|c|}
\hline Variable & $\mathrm{CON}$ & ALC & ES & $\mathrm{F}$ & p-value \\
\hline \multicolumn{6}{|l|}{ Step 1: Encoding } \\
\hline \multirow[t]{2}{*}{ Average Encoding } & $4.25(0.79)$ & $3.68(1.07)$ & 0.61 & 2.67 & 0.109 \\
\hline & & & age & 11.31 & 0.002 \\
\hline \multicolumn{6}{|l|}{ Step 2: Attribution } \\
\hline Hostile Attribution & $0.43(0.28)$ & $0.55(0.30)$ & 0.41 & 2.06 & 0.157 \\
\hline Non-hostile Attribution & $0.25(0.24)$ & $0.27(0.28)$ & 0.08 & 0.07 & 0.793 \\
\hline \multicolumn{6}{|l|}{ Step 3: Goals } \\
\hline Proportion Pro-social & $0.92(0.12)$ & $0.79(0.31)$ & 0.55 & 3.85 & 0.055 \\
\hline Proportion Negative/Aggressive & $0.03(0.07)$ & $0.06(0.15)$ & 0.26 & 1.16 & 0.287 \\
\hline Proportion Nonsocial & $0.04(0.10)$ & $0.07(0.12)$ & 0.27 & 0.94 & 0.337 \\
\hline Proportion Inept & $0.00(0.00)$ & $0.05(0.11)$ & 0.64 & 5.66 & 0.021 \\
\hline Proportion Irrelevant & $0.01(0.03)$ & $0.02(0.05)$ & 0.24 & 1.10 & 0.300 \\
\hline \multicolumn{6}{|l|}{ Step 4: Response Generation } \\
\hline \multirow[t]{2}{*}{ Number of Total Responses } & $31.12(16.04)$ & $26.65(14.16)$ & 0.30 & 0.45 & 0.504 \\
\hline & & & age & 3.30 & 0.075 \\
\hline Proportion Competent & $0.71(0.17)$ & $0.64(0.25)$ & 0.33 & 1.13 & 0.293 \\
\hline Proportion Aggressive & $0.04(0.06)$ & $0.05(0.09)$ & 0.13 & 0.58 & 0.451 \\
\hline Proportion Inept & $0.24(0.14)$ & $0.26(0.20)$ & 0.12 & 0.13 & 0.718 \\
\hline \multirow[t]{2}{*}{ Number Non-repeated Responses } & $23.69(11.48)$ & $21.19(10.64)$ & 0.23 & 0.06 & 0.807 \\
\hline & & & age & 7.98 & 0.007 \\
\hline Proportion Non-repeated Competent & $0.68(0.15)$ & $0.62(0.24)$ & 0.30 & 1.13 & 0.292 \\
\hline Proportion Non-repeated Aggressive & $0.04(0.06)$ & $0.06(0.08)$ & 0.28 & 0.64 & 0.428 \\
\hline Proportion Non-repeated Inept & $0.26(0.14)$ & $0.28(0.20)$ & 0.12 & 0.11 & 0.739 \\
\hline \multirow[t]{2}{*}{ Proportion First Competent } & $0.82(0.18)$ & $0.67(0.25)$ & 0.69 & 3.96 & 0.052 \\
\hline & & & age & 7.62 & 0.008 \\
\hline Proportion First Aggressive & $0.01(0.05)$ & $0.05(0.07)$ & 0.66 & 4.10 & 0.048 \\
\hline \multirow[t]{2}{*}{ Proportion First Inept } & $0.12(0.14)$ & $0.21(0.22)$ & 0.49 & 1.30 & 0.260 \\
\hline & & & age & 4.43 & 0.041 \\
\hline \multicolumn{6}{|l|}{ Step 5: Response Evaluation } \\
\hline \multirow[t]{2}{*}{ Affiliation Competent } & $0.18(0.05)$ & $0.21(0.07)$ & 0.49 & 3.66 & 0.062 \\
\hline & & & $\operatorname{sex}$ & 4.69 & 0.035 \\
\hline Affiliation Aggressive & $0.44(0.03)$ & $0.43(0.05)$ & 0.24 & 1.01 & 0.319 \\
\hline Affiliation Inept & $0.38(0.03)$ & $0.36(0.04)$ & 0.57 & 3.81 & 0.057 \\
\hline \multirow[t]{2}{*}{ Instrumental Competent } & $0.18(0.05)$ & $0.23(0.06)$ & 0.91 & 6.70 & 0.013 \\
\hline & & & age & 2.52 & 0.119 \\
\hline Instrumental Aggressive & $0.44(0.03)$ & $0.42(0.05)$ & 0.49 & 3.14 & 0.083 \\
\hline Instrumental Inept & $0.38(0.03)$ & $0.36(0.04)$ & 0.57 & 6.93 & 0.011 \\
\hline \multicolumn{6}{|l|}{ Step 6: Enactment } \\
\hline Average Enactment & $2.72(0.57)$ & $2.59(0.37)$ & 0.27 & 0.85 & 0.360 \\
\hline
\end{tabular}


lower proportion of competent responses for their first response. Children in the ALC group also evaluated competent responses as less effective than controls in convincing the other children to let them play and viewed inept responses as more effective in achieving this goal. Alcohol-exposed children also evaluated inept responses more favorably than controls in getting the other children to like them. A medium to large effect was also found on encoding with the ALC group encoding and recalling less relevant information than the CON group; however, this difference was not significant when age was included in the model.

Provocation Group differences were observed in the encoding, attribution, response evaluation, and enactment steps of social information processing (see Table 4). Specifically, children in the ALC group encoded and recalled less relevant information from the vignettes than controls. On the attribution step, alcohol-exposed children were less likely than their typically developing peers to
Table 4 Means, Standard Deviations, Effect Sizes, and Significance Testing Results for Non-exposed Controls (CON) and Children with Prenatal Alcohol Exposure (ALC) on Social Information Processing Variables in Provocation Situations

\begin{tabular}{|c|c|c|c|c|c|}
\hline Variable & $\mathrm{CON}$ & ALC & ES & $\mathrm{F}$ & p-value \\
\hline \multicolumn{6}{|l|}{ Step 1: Encoding } \\
\hline \multirow[t]{2}{*}{ Average Encoding } & $4.82(0.38)$ & $4.33(0.66)$ & 0.91 & 7.73 & 0.008 \\
\hline & & & age & 5.49 & 0.023 \\
\hline \multicolumn{6}{|l|}{ Step 2: Attribution } \\
\hline Hostile Attribution & $0.41(0.27)$ & $0.43(0.25)$ & 0.08 & 0.07 & 0.791 \\
\hline Non-hostile Attribution & $0.12(0.15)$ & $0.06(0.08)$ & 0.50 & 3.75 & 0.058 \\
\hline \multicolumn{6}{|l|}{ Step 3: Goals } \\
\hline \multirow[t]{2}{*}{ Proportion Pro-social } & $0.89(0.22)$ & $0.80(0.29)$ & 0.35 & 0.67 & 0.416 \\
\hline & & & age & 3.31 & 0.075 \\
\hline Proportion Negative/Aggressive & $0.04(0.08)$ & $0.04(0.07)$ & 0.00 & 0.04 & 0.842 \\
\hline Proportion Nonsocial & $0.03(0.10)$ & $0.05(0.12)$ & 0.18 & 0.71 & 0.405 \\
\hline Proportion Inept & $0.04(0.16)$ & $0.08(0.18)$ & 0.23 & 0.54 & 0.465 \\
\hline Proportion Irrelevant & $0.00(0.02)$ & $0.03(0.08)$ & 0.51 & 1.77 & 0.189 \\
\hline \multicolumn{6}{|l|}{ Step 4: Response Generation } \\
\hline \multirow[t]{2}{*}{ Number of Total Responses } & $32.27(15.82)$ & $26.15(13.92)$ & 0.41 & 1.01 & 0.319 \\
\hline & & & age & 5.53 & 0.023 \\
\hline Proportion Competent & $0.81(0.18)$ & $0.77(0.22)$ & 0.20 & 0.67 & 0.418 \\
\hline Proportion Aggressive & $0.08(0.12)$ & $0.07(0.15)$ & 0.07 & 0.05 & 0.817 \\
\hline Proportion Inept & $0.11(0.13)$ & $0.16(0.20)$ & 0.30 & 1.44 & 0.236 \\
\hline \multirow[t]{2}{*}{ Number Non-repeated Responses } & $26.65(12.15)$ & $22.50(11.24)$ & 0.35 & 0.50 & 0.483 \\
\hline & & & age & 8.89 & 0.004 \\
\hline Proportion Non-repeated Competent & $0.81(0.18)$ & $0.75(0.23)$ & 0.29 & 0.87 & 0.355 \\
\hline Proportion Non-repeated Aggressive & $0.08(0.12)$ & $0.07(0.16)$ & 0.07 & 0.06 & 0.810 \\
\hline Proportion Non-repeated Inept & $0.11(0.13)$ & $0.17(0.20)$ & 0.36 & 1.81 & 0.185 \\
\hline \multirow[t]{2}{*}{ Proportion First Competent } & $0.85(0.21)$ & $0.77(0.24)$ & 0.35 & 0.58 & 0.449 \\
\hline & & & age & 7.01 & 0.011 \\
\hline Proportion First Aggressive & $0.04(0.10)$ & $0.05(0.16)$ & 0.07 & 0.12 & 0.727 \\
\hline \multirow[t]{2}{*}{ Proportion First Inept } & $0.09(0.17)$ & $0.15(0.20)$ & 0.32 & 0.54 & 0.467 \\
\hline & & & age & 4.19 & 0.046 \\
\hline \multicolumn{6}{|l|}{ Step 5: Response Evaluation } \\
\hline Affiliation Competent & $0.25(0.04)$ & $0.28(0.04)$ & 0.75 & 6.17 & 0.016 \\
\hline Affiliation Aggressive & $0.39(0.03)$ & $0.38(0.03)$ & 0.33 & 2.40 & 0.128 \\
\hline Affiliation Inept & $0.35(0.03)$ & $0.34(0.03)$ & 0.33 & 2.80 & 0.100 \\
\hline Instrumental Competent & $0.26(0.04)$ & $0.27(0.04)$ & 0.25 & 1.84 & 0.181 \\
\hline Instrumental Aggressive & $0.40(0.04)$ & $0.39(0.04)$ & 0.25 & 0.87 & 0.356 \\
\hline Instrumental Inept & $0.35(0.04)$ & $0.34(0.04)$ & 0.25 & 0.16 & 0.696 \\
\hline \multicolumn{6}{|l|}{ Step 6: Enactment } \\
\hline Average Enactment & $2.72(0.53)$ & $2.29(0.38)$ & 0.93 & 11.27 & 0.002 \\
\hline
\end{tabular}


Table 5 Means, Standard Deviations, Effect Sizes, and Significance Testing Results for Non-exposed Controls (CON) and Children with Prenatal Alcohol Exposure (ALC) on the Test of Problem Solving, $3 \mathrm{rd}$ Edition

\begin{tabular}{lcllrl}
\hline Variable & CON & ALC & ES & F & p-value \\
\hline Making Inferences & $100.42(10.09)$ & $92.08(16.20)$ & 0.62 & 4.97 & 0.030 \\
Sequencing & $101.00(9.49)$ & $87.46(17.28)$ & 0.97 & 12.26 & 0.001 \\
Negative Questions & $99.62(12.29)$ & $84.19(17.83)$ & 1.01 & 13.19 & 0.001 \\
Problem Solving & $100.54(11.37)$ & $90.15(15.88)$ & 0.75 & 7.35 & 0.009 \\
Predicting & $97.85(11.74)$ & $87.62(15.62)$ & 0.74 & 7.13 & 0.010 \\
Determining Causes & $100.88(13.06)$ & $88.69(14.38)$ & 0.89 & 10.24 & 0.002 \\
Total Test & $100.12(9.34)$ & $86.58(16.99)$ & 0.99 & 12.68 & 0.001 \\
\hline
\end{tabular}

attribute benign intent when the intent of the other child was hostile or ambiguous. Children with prenatal alcohol exposure evaluated competent responses as less effective in getting the other child to like them on the response evaluation step. Finally, control children were rated more effective in their ability to repeat a competent response with appropriate eye contact and tone of voice during the enactment step.

Concurrent Validity: Test of Problem Solving-Third Edition

Group Differences Group differences were statistically significant for all scales of the TOPS-3 with medium and large effect sizes (see Table 5). For all scales, the ALC group had lower scores than controls, reflecting weaker critical thinking and problem solving skills.

Correlational Analyses Canonical correlation analyses were conducted within each social information processing domain separately. See Table 6 for model fit statistics for both domains and Table 7 for standardized canonical coefficients and canonical loadings.

Canonical correlation analyses for both domains produced one significant canonical variate pair. Encoding, total number of responses, and the proportion of first competent responses loaded highly on the social information processing variate for both domains. The social information

Table 6 Canonical Correlation and Fit Indices for the Relationship between Social Information Processing and the Test of Problem Solving, 3rd Edition

\begin{tabular}{lllrll}
\hline Domain & $\mathrm{r}_{\mathrm{c}}$ & Wilks $\lambda$ & \multicolumn{1}{c}{$\chi^{2}$} & $\mathrm{p}$ value & $\eta 2$ \\
\hline Group Entry & 0.708 & 0.386 & 43.31 & 0.009 & 0.614 \\
& 0.407 & 0.774 & 11.64 & 0.706 & 0.226 \\
& 0.252 & 0.928 & 3.38 & 0.908 & 0.072 \\
& 0.093 & 0.991 & 0.40 & 0.941 & 0.009 \\
Provocation & 0.628 & 0.430 & 38.80 & 0.003 & 0.570 \\
& 0.445 & 0.710 & 15.76 & 0.107 & 0.290 \\
& 0.339 & 0.885 & 5.63 & 0.229 & 0.115 \\
\hline
\end{tabular}

processing variate correlated highly with the problem solving variate formed from TOPS-3 variables, suggesting that the encoding and response generation steps are most strongly related to TOPS-3 performance. Results indicate that weaker social information processing skills are associated with poorer critical thinking and problem solving skills. Taken together, strong correlations with the TOPS-3 for both domains provide evidence for the concurrent validity of the social information processing measure.

Supplemental Analyses for Social Information Processing Variables

Intellectual Functioning Comparison of group means revealed that while some of the differences observed with the larger sample were likely due to IQ, some deficits remained (see Table 8). Specifically, group differences seen on the encoding (Provocation), goal (Group Entry), and response evaluation (Group Entry and Provocation) steps were attenuated when controlling for IQ. In contrast, medium to large effects remained on the aggressive first response generation variable on Group Entry, and the attribution and enactment steps on Provocation.

FAS Diagnosis Subgroup differences were similar across Group Entry and Provocation domains. Children with FAS tended to have lower mean scores than alcohol-exposed children without FAS on encoding, prosocial goals, and total number of generated responses. On the response generation step, children with FAS tended to give more inept responses, whereas alcohol-exposed children without FAS tended to give more aggressive responses. Both alcohol-exposed groups had similar enactment scores (see Table 9).

\section{Discussion}

The aim of the current study was to assess the social information processing skills of school-age children with histories of heavy prenatal alcohol exposure. Our findings corroborate and augment caregiver reported social deficits 
Table 7 Standardized Canonical Coefficients (coeff), Canonical Loadings (load), and Adequacy (adeq) and Redundancy (redun) Coefficients for Canonical Correlation Analyses Between Social Information Processing and the Test of Problem Solving, 3rd Edition

\begin{tabular}{|c|c|c|c|c|c|c|c|c|c|}
\hline Variables & coeff & load & adeq & redun & Related Constructs & coeff & load & adeq & redun \\
\hline Group Entry & & & 0.486 & 0.244 & & & & 0.702 & 0.352 \\
\hline Encoding & -0.12 & -0.51 & & & Making Inferences & 0.10 & -0.77 & & \\
\hline Nonhostile Attribution & 0.61 & 0.88 & & & Sequencing & -0.25 & -0.87 & & \\
\hline First Competent & -0.45 & -0.80 & & & Negative Questions & 0.18 & -0.81 & & \\
\hline \multirow[t]{3}{*}{ Total Responses } & -0.08 & -0.52 & & & Problem Solving & -0.30 & -0.86 & & \\
\hline & & & & & Predicting & -0.59 & -0.94 & & \\
\hline & & & & & Determining Causes & -0.25 & -0.77 & & \\
\hline Provocation & & & 0.610 & 0.240 & & & & 0.676 & 0.266 \\
\hline Encoding & -0.68 & -0.93 & & & Making Inferences & -0.55 & -0.92 & & \\
\hline First Competent & -0.24 & -0.75 & & & Sequencing & 0.19 & -0.75 & & \\
\hline \multirow[t]{4}{*}{ Total Responses } & -0.30 & -0.64 & & & Negative Questions & -0.01 & -0.78 & & \\
\hline & & & & & Problem Solving & -0.01 & -0.83 & & \\
\hline & & & & & Predicting & -0.47 & -0.90 & & \\
\hline & & & & & Determining Causes & -0.28 & -0.74 & & \\
\hline
\end{tabular}

in this population by providing more direct measurement of children's social cognition, and provide insight into possible mechanisms for impaired social functioning. Children with prenatal alcohol exposure demonstrated difficulties in social information processing on all six steps of Crick and Dodge's reformulated model (Crick and Dodge 1994), across both hypothetical Group Entry and Provocation situations. However, areas of difficulty differed by the type of social situation (i.e., Group Entry vs. Provocation), which is consistent with the previously reported situational specificity of social information processing (Dodge et al. 2002). Specifically, in hypothetical Group Entry situations, children with heavy prenatal alcohol exposure exhibited less skillful social information processing patterns than controls in the goal selection, response generation, and response evaluation steps. In Provocation situations, children with prenatal alcohol exposure exhibited difficulties in the encoding, attribution, response evaluation, and enactment steps of social information processing.

Another goal of the current study was to provide evidence of concurrent validity of the social information processing measure in this population. On a measure of critical thinking and problem solving skills (TOPS-3), children with prenatal alcohol exposure demonstrated impaired performance in comparison to non-exposed controls across all subscales. Canonical correlation analyses revealed large and positive correlations between social information processing and TOPS-3 performance. While the measures exhibited strong correlations of .71 and .63 for the Group Entry and Provocation domains, respectively, they are not redundant and each provides unique information. The TOPS-3 places larger emphasis on language skills than does the social information processing measure, which may account for the larger effect sizes on the TOPS-3.

Multiple studies using caregiver-report measures have identified impairments in social functioning in children with prenatal alcohol exposure, with interpersonal relationship skills being the most problematic (Thomas et al. 1998; Whaley et al. 2001). The social information processing patterns of children with prenatal alcohol exposure reported herein suggest possible mechanisms for observed social difficulties that are common in this population. For example, children who do not encode sufficient or appropriate social cues or misinterpret peers' intent may ignore or respond negatively to peers' attempts to interact. Similarly, alcohol-exposed children who evaluate inept responses more favorably may select inappropriate or ineffective strategies for enactment. Deficits in social functioning are likely to become more apparent as children get older and expectations increase (Thomas et al. 1998; Whaley et al. 2001). Our findings are also consistent with self-reported difficulties in social problem solving in adolescents with prenatal alcohol exposure (McGee et al. 2008), suggesting that difficulties persist and indicating the need for early intervention.

It is important to consider whether children with prenatal alcohol exposure exhibit a general social information processing deficit or if difficulties are more situationally based. Results from the TOPS-3 suggest some general deficiencies in social problem solving in this population. However, when social information processing was examined in the context of two specific social situations, areas of difficulty varied as a function of the situation. Specific maladaptive processing patterns emerged within Group Entry and Provocation domains, but only the response 
Table 8 Means and Effect Sizes for IQ-matched Non-exposed Controls (CON) and Children with Prenatal Alcohol Exposure (ALC) on Social Information Processing Variables in Group Entry and Provocation Situations

\begin{tabular}{|c|c|c|c|c|c|c|}
\hline \multirow[t]{2}{*}{ Variable } & \multicolumn{3}{|l|}{ Group Entry } & \multicolumn{3}{|l|}{ Provocation } \\
\hline & $\mathrm{CON}$ & ALC & ES & $\mathrm{CON}$ & ALC & ES \\
\hline \multicolumn{7}{|l|}{ Step 1: Encoding } \\
\hline Average Encoding & $4.06(1.01)$ & $3.99(1.22)$ & 0.06 & $4.67(0.55)$ & $4.53(0.75)$ & 0.21 \\
\hline \multicolumn{7}{|l|}{ Step 2: Attribution } \\
\hline Hostile Attribution & $0.30(0.25)$ & $0.47(0.23)$ & 0.71 & $0.40(0.32)$ & $0.38(0.19)$ & 0.08 \\
\hline Non-hostile Attribution & $0.35(0.27)$ & $0.20(0.15)$ & 0.69 & $0.12(0.19)$ & $0.03(0.07)$ & 0.63 \\
\hline \multicolumn{7}{|l|}{ Step 3: Goals } \\
\hline Proportion Prosocial & $0.86(0.16)$ & $0.92(0.17)$ & 0.36 & $0.77(0.30)$ & $0.97(0.05)$ & 0.93 \\
\hline Prop. Negative/Aggressive & $0.03(0.07)$ & $0.06(0.18)$ & 0.22 & $0.07(0.11)$ & $0.02(0.05)$ & 0.59 \\
\hline Proportion Nonsocial & $0.10(0.14)$ & $0.02(0.05)$ & 0.76 & $0.07(0.15)$ & $0.00(0.00)$ & 0.66 \\
\hline Proportion Inept & $0.00(0.00)$ & $0.00(0.00)$ & 0.00 & $0.10(0.25)$ & $0.01(0.04)$ & 0.50 \\
\hline Proportion Irrelevant & $0.01(0.04)$ & $0.00(0.00)$ & 0.35 & $0.00(0.00)$ & $0.00(0.00)$ & 0.00 \\
\hline \multicolumn{7}{|l|}{ Step 4: Response Generation } \\
\hline Number of Total Responses & $24.30(16.52)$ & $27.10(11.07)$ & 0.20 & $26.10(16.65)$ & $27.40(10.63)$ & 0.09 \\
\hline Proportion Competent & $0.70(0.19)$ & $0.74(0.24)$ & 0.18 & $0.75(0.25)$ & $0.81(0.24)$ & 0.24 \\
\hline Proportion Aggressive & $0.04(0.08)$ & $0.06(0.11)$ & 0.21 & $0.11(0.18)$ & $0.11(0.24)$ & 0.00 \\
\hline Proportion Inept & $0.23(0.17)$ & $0.17(0.17)$ & 0.35 & $0.14(0.18)$ & $0.08(0.14)$ & 0.37 \\
\hline \# Non-repeated Responses & $17.70(0.76)$ & $21.00(7.85)$ & 0.59 & $20.60(9.72)$ & $23.30(7.51)$ & 0.31 \\
\hline Prop. Non-repeated Competent & $0.68(0.16)$ & $0.72(0.24)$ & 0.20 & $0.75(0.25)$ & $0.79(0.24)$ & 0.16 \\
\hline Prop. Non-repeated Aggressive & $0.04(0.08)$ & $0.06(0.11)$ & 0.21 & $0.11(0.18)$ & $0.11(0.24)$ & 0.00 \\
\hline Proportion Non-repeated Inept & $0.23(0.16)$ & $0.19(0.18)$ & 0.23 & $0.14(0.17)$ & $0.10(0.15)$ & 0.25 \\
\hline Proportion First Competent & $0.72(0.20)$ & $0.78(0.22)$ & 0.29 & $0.72(0.30)$ & $0.81(0.25)$ & 0.33 \\
\hline Proportion First Aggressive & $0.02(0.07)$ & $0.04(0.08)$ & 0.27 & $0.07(0.14)$ & $0.10(0.25)$ & 0.15 \\
\hline Proportion First Inept & $0.16(0.16)$ & $0.13(0.16)$ & 0.19 & $0.18(0.25)$ & $0.08(0.15)$ & 0.49 \\
\hline \multicolumn{7}{|l|}{ Step 5: Response Evaluation } \\
\hline Affiliation Competent & $0.20(0.05)$ & $0.19(0.05)$ & 0.20 & $0.27(0.04)$ & $0.27(0.04)$ & 0.00 \\
\hline Affiliation Aggressive & $0.44(0.03)$ & $0.44(0.04)$ & 0.00 & $0.39(0.03)$ & $0.39(0.02)$ & 0.00 \\
\hline Affiliation Inept & $0.37(0.04)$ & $0.37(0.03)$ & 0.00 & $0.34(0.03)$ & $0.34(0.04)$ & 0.00 \\
\hline Instrumental Competent & $0.20(0.05)$ & $0.20(0.04)$ & 0.00 & $0.26(0.03)$ & $0.26(0.04)$ & 0.00 \\
\hline Instrumental Aggressive & $0.43(0.03)$ & $0.43(0.04)$ & 0.00 & $0.41(0.04)$ & $0.39(0.03)$ & 0.57 \\
\hline Instrumental Inept & $0.37(0.04)$ & $0.37(0.04)$ & 0.00 & $0.33(0.04)$ & $0.34(0.05)$ & 0.22 \\
\hline \multicolumn{7}{|l|}{ Step 6: Enactment } \\
\hline Average Enactment & $2.70(0.61)$ & $2.49(0.33)$ & 0.43 & $2.71(0.47)$ & $2.14(0.17)$ & 1.61 \\
\hline
\end{tabular}

evaluation step was problematic for alcohol-exposed children across both types of situations. Examination of cognition in other types of social situations will assist in determining if specific difficulties, such as our response evaluation finding, are consistent across situations, as well as identifying which steps are particularly problematic in specific social situations.

The influences of several potential moderating demographic variables on social information processing were also assessed in the current study. Significant age effects were found for the encoding and response generation steps across social domains, consistent with previous studies (Gifford-Smith and Rabiner 2004). As age increased, children in both groups recalled more relevant cues and generated a larger number of total responses and a higher proportion of competent first responses. While there was a significant difference between groups in their abilities to encode and recall relevant cues in Provocation situations, for Group Entry situations, the difference in encoding ability was no longer significant when age was included in the model. Age may play a larger role in Group Entry situations since cues may be more subtle and less emotionally salient in comparison to Provocation situations. Therefore, younger children may have a harder time discerning these cues than older children. In contrast, sex was only significantly related to the evaluation of compe- 
Table 9 Means and Effect Sizes for Children with Prenatal Alcohol Exposure with (FAS) and without (PEA) FAS on Social Information Processing Variables in Group Entry and Provocation Situations

\begin{tabular}{|c|c|c|c|c|c|c|c|}
\hline Demographics & FAS & PEA & \multicolumn{2}{|l|}{ Demographics } & FAS & \multicolumn{2}{|l|}{ PEA } \\
\hline \multirow[t]{2}{*}{ Age $[$ Mean $(\mathrm{SD})$} & $\begin{array}{l}6 \\
9.01(1.40)\end{array}$ & $\begin{array}{l}18 \\
9.63(1.71)\end{array}$ & \multicolumn{2}{|l|}{ Sex $[N(\%$ Female $)]$} & $\begin{array}{l}2(33.3) \\
81.83(20.48)\end{array}$ & \multicolumn{2}{|l|}{$9(50.0)$} \\
\hline & & Group Entry & & & Provocation & & \\
\hline \multicolumn{2}{|l|}{ Variable } & FAS & PEA & ES & FAS & PEA & ES \\
\hline \multicolumn{8}{|l|}{ Step 1: Encoding } \\
\hline \multicolumn{2}{|c|}{ Average Encoding } & $3.46(0.76)$ & $3.94(1.03)$ & 0.53 & $3.89(0.62)$ & $4.54(0.61)$ & 1.06 \\
\hline \multicolumn{8}{|c|}{ Step 2: Attribution } \\
\hline \multicolumn{2}{|c|}{ Hostile Attribution } & $0.53(0.30)$ & $0.60(0.28)$ & 0.24 & $0.47(0.34)$ & $0.44(0.23)$ & 0.10 \\
\hline \multicolumn{2}{|c|}{ Non-hostile Attribution } & $0.44(0.37)$ & $0.16(0.14)$ & 1.00 & $0.09(0.09)$ & $0.06(0.08)$ & 0.35 \\
\hline \multicolumn{8}{|l|}{ Step 3: Goals } \\
\hline \multicolumn{2}{|c|}{ Proportion Prosocial } & $0.78(0.37)$ & $0.87(0.21)$ & 0.30 & $0.60(0.36)$ & $0.93(0.14)$ & 1.21 \\
\hline \multicolumn{2}{|c|}{ Proportion Negative/Aggressive } & $0.06(0.14)$ & $0.05(0.15)$ & 0.07 & $0.07(0.09)$ & $0.02(0.06)$ & 0.65 \\
\hline \multicolumn{2}{|c|}{ Proportion Nonsocial } & $0.07(0.13)$ & $0.04(0.08)$ & 0.28 & $0.02(0.05)$ & $0.03(0.07)$ & 0.16 \\
\hline \multicolumn{2}{|c|}{ Proportion Inept } & $0.06(0.09)$ & $0.03(0.07)$ & 0.37 & $0.26(0.30)$ & $0.01(0.04)$ & 1.17 \\
\hline \multicolumn{2}{|c|}{ Proportion Irrelevant } & $0.04(0.06)$ & $0.01(0.03)$ & 0.63 & $0.06(0.14)$ & $0.01(0.03)$ & 0.49 \\
\hline \multicolumn{8}{|c|}{ Step 4: Response Generation } \\
\hline \multicolumn{2}{|c|}{ Number of Total Responses } & $19.83(9.83)$ & $30.28(14.74)$ & 0.83 & $20.67(8.38)$ & $29.67(14.62)$ & 0.76 \\
\hline \multicolumn{2}{|c|}{ Proportion Competent } & $0.58(0.31)$ & $0.66(0.24)$ & 0.29 & $0.77(0.26)$ & $0.80(0.21)$ & 0.13 \\
\hline \multicolumn{2}{|c|}{ Proportion Aggressive } & $0.02(0.04)$ & $0.06(0.10)$ & 0.53 & $0.03(0.05)$ & $0.09(0.18)$ & 0.45 \\
\hline \multicolumn{2}{|c|}{ Proportion Inept } & $0.32(0.28)$ & $0.24(0.19)$ & 0.33 & $0.20(0.25)$ & $0.11(0.14)$ & 0.44 \\
\hline \multicolumn{2}{|c|}{ \# Non-repeated Responses } & $16.33(6.89)$ & $23.83(11.22)$ & 0.81 & $17.83(6.46)$ & $25.44(11.74)$ & 0.80 \\
\hline \multicolumn{2}{|c|}{ Prop. Non-repeated Competent } & $0.55(0.30)$ & $0.65(0.24)$ & 0.37 & $0.77(0.27)$ & $0.78(0.21)$ & 0.04 \\
\hline \multicolumn{2}{|c|}{ Prop. Non-repeated Aggressive } & $0.03(0.04)$ & $0.06(0.10)$ & 0.39 & $0.03(0.06)$ & $0.10(0.18)$ & 0.52 \\
\hline Proportion Nol & ted Inept & $0.35(0.27)$ & $0.25(0.18)$ & 0.44 & $0.20(0.25)$ & $0.13(0.15)$ & 0.34 \\
\hline Proportion Firs & petent & $0.61(0.34)$ & $0.72(0.22)$ & 0.38 & $0.74(0.28)$ & $0.81(0.22)$ & 0.28 \\
\hline Proportion Firs & essive & $0.02(0.06)$ & $0.05(0.08)$ & 0.42 & $0.04(0.06)$ & $0.06(0.19)$ & 0.14 \\
\hline Proportion Firs & & $0.30(0.34)$ & $0.18(0.18)$ & 0.44 & $0.22(0.26)$ & $0.10(0.16)$ & 0.56 \\
\hline Step 5: Respons & ation & & & & & & \\
\hline Affiliation Cor & & $0.25(0.08)$ & $0.20(0.07)$ & 0.67 & $0.29(0.03)$ & $0.27(0.04)$ & 0.57 \\
\hline Affiliation Agg & & $0.41(0.07)$ & $0.43(0.05)$ & 0.33 & $0.37(0.03)$ & $0.38(0.03)$ & 0.33 \\
\hline Affiliation Iner & & $0.34(0.05)$ & $0.37(0.03)$ & 0.73 & $0.34(0.02)$ & $0.34(0.03)$ & 0.00 \\
\hline Instrumental C & & $0.27(0.06)$ & $0.21(0.06)$ & 1.00 & $0.28(0.04)$ & $0.27(0.04)$ & 0.25 \\
\hline Instrumental $\mathrm{A}$ & & $0.40(0.07)$ & $0.42(0.04)$ & 0.35 & $0.39(0.05)$ & $0.39(0.04)$ & 0.00 \\
\hline Instrumental In & & $0.33(0.04)$ & $0.37(0.03)$ & 1.13 & $0.34(0.04)$ & $0.34(0.05)$ & 0.00 \\
\hline Step 6: Enactme & & & & & & & \\
\hline Average Enact & & $2.67(0.46)$ & $2.61(0.36)$ & 0.15 & $2.26(0.55)$ & $2.28(0.35)$ & 0.04 \\
\hline
\end{tabular}

tent responses in obtaining an affiliation goal for Group Entry situations. On this variable, girls in both groups evaluated competent responses as more effective than did boys. Racial and socioeconomic backgrounds were not significantly related to social information processing variables.

Supplementary analyses were also conducted on the impact of intellectual functioning and FAS diagnosis on social information processing variables. Consistent with previous studies on social functioning in children with FASD (Thomas et al. 1998), some difficulties in social information processing were above and beyond what would be predicted by intellectual functioning, especially for more behaviorally oriented variables such as aggressive response generation and behavioral enactment. Some subgroup differences were also identified in alcohol-exposed children. Children with FAS demonstrated less effective social 
information processing on encoding, goal, and response generation steps and were more likely to provide inept responses, whereas alcohol-exposed children without FAS tended to give more aggressive responses. Some subgroup differences may be related to lower IQ scores in the FAS subgroup. Children with FAS are more likely to be diagnosed earlier and receive services than those without FAS, which may result in more structured environments, caregiver attributions focusing on the alcohol exposure rather than behavior being intentional, and fewer disruptive behavior problems. These results should be interpreted cautiously due to small sample sizes.

Maladaptive patterns of processing social information have been linked to emotional and behavioral problems as well as poor peer interactions (e.g., Dodge et al. 2002; Dodge et al. 2003). Consequently, children who exhibit these patterns of processing social information are more likely to be disliked and rejected by peers, which may result in fewer opportunities to acquire and practice social skills and could lead to further maladjustment and rejection (Dodge et al. 2003; Gifford-Smith and Rabiner 2004; Kupersmidt and DeRosier 2004). Social difficulties are a life long problem for individuals with prenatal alcohol exposure and include high rates of social withdrawal, loneliness, teasing or bullying, antisocial behavior, dependency, and difficulty with employment (Streissguth et al 1996).

Cognitive and behavioral impairments common in children with prenatal alcohol exposure likely contribute to difficulties with social information processing. For example, deficits in intellectual functioning may lead to weaker reasoning ability and biased interpretations. With respect to language, one preliminary study suggested that individuals with FASD have more difficulty providing information to communicative partners (Coggins et al. 1998). Similarly, children with specific language impairments often exhibit difficulties with social functioning, which likely reflects an interaction between language limitations, social context, and biases people associate with limited verbal proficiency (Redmond and Rice 1998). Learning and memory deficits are likely to lead to more difficulty encoding relevant cues, learning from previous experiences, and recalling consequences of actions. Impairments in executive functioning may also lead to difficulty generating multiple responses, inhibiting acting on the first response generated, and holding and manipulating multiple cues or responses in mind during interpretation or response evaluation. Preliminary analyses (unpublished data) with the current sample are consistent with this hypothesis; caregiver-report measures of executive functioning were significantly correlated with social information processing. Similarly, disruptive behavior problems were related to less skillful social information processing. Future studies should directly evaluate the influences of cognition and behavior on social information processing.

There are several additional limitations to the current study. Statistical analyses were limited by the large number of variables and their psychometric properties. While univariate ANOVA procedures are robust to violations to normality (Maxwell and Delaney 2004), the large number of comparisons required for this study increased the probability of committing a type one error. In an attempt to reduce this error, significance test results were only considered meaningful if differences represented a medium effect or larger. Canonical correlation analyses depend heavily on the variables included in the model. To improve power and minimize multicolinearity, social information processing variables were only selected if correlations with the TOPS3 were between .3 and .7. Due to sample size limitations, canonical analyses should be interpreted cautiously.

Since social cognitive and behavioral competencies improve dramatically with age and the types of relevant social situations vary across age groups, the age range for this study was limited to children between the ages of seven and 11 . Thus, the findings from the current study may not be generalizable to other age groups, such as preschool children or adolescents. Future studies should examine social information processing patterns of alcohol-exposed individuals of varying age groups. While Group Entry and Provocation situations are common social problems faced by elementary school children, children may face many other types of social situations, and research suggests that performance may vary significantly across different situational contexts (Dodge et al. 2002). Additional studies should include a wide range of social situations to examine additional contextual factors related to social information processing.

Another limitation of the current study is the difference in home placement between groups. It is possible that the observed deficits are more related to the experience of being removed from the biological family than the alcohol exposure per se. While sample sizes were small, scatter plots did not reveal any meaningful trends in the alcohol-exposed group with respect to home environment. In addition, the length of time in children's current placement was not significantly related to social information processing variables. Future studies including a comparison group of children without alcohol exposure matched on the type and length in the home environment may help resolve this issue. Prospective designs of prenatal alcohol exposure may also be useful in addressing this question as children typically reside with their biological families. However, children tend to be less affected and deficits may be more subtle.

In spite of these limitations, this is the first study to comprehensively assess the social information processing skills of school-age children with prenatal alcohol expo- 
sure. Future work can build on the current findings and improve our understanding of social outcomes in children with histories of prenatal alcohol exposure by identifying risk and protective factors and using other methodologies, such as direct observation in naturalistic settings. Results from the current study emphasize the need for early intervention and provide insight on possible mechanisms for observed difficulties in social functioning. Social skills are amenable to intervention in this population (O'Connor et al. 2006), and direct instruction in social information processing skills may help children generalize and maintain intervention gains. As demonstrated by the current study, difficulties in processing social information vary by situation type, and therefore interventions should target multiple social situations and contextual variability. If interventions are begun early, these difficulties in processing may be ameliorated or lessened before the child experiences considerable social rejection and maladjustment in later stages of life.

Acknowledgments The authors thank Drs. Mary O'Connor and Lyn Laboriel for their assistance with recruitment, the members of the Center for Behavioral Teratology, and the families who graciously participate in our studies. Supported by NIAAA grants F31 AA016047, T32 AA013525, and R01 AA010417.

Open Access This article is distributed under the terms of the Creative Commons Attribution Noncommercial License which permits any noncommercial use, distribution, and reproduction in any medium, provided the original author(s) and source are credited.

\section{Appendix: Social Information Processing Interview Example Responses}

Encoding: Responses were coded according to the number of details provided by the child for a possible total of 6 points. Children received 2 points if they described the actions of the other child toward one's self (e.g., they smiled at me, they moved away from me, he spilled my paint), and 1 point for each of the following: action of self (e.g., walked up), identification of activity, mention the presence of other child or children, and any detail about the setting (e.g., at a picnic table). The actions of the other child (ren) were given greater weighting in scoring due to the significance of cues.

Goal Clarification: For Group Entry situations, examples of prosocial goals include asking or being invited to play, making an assertive statement about the other children's response, or the other children saying they are sorry. In Provocation situations, examples of prosocial goals include wanting the other child to apologize or fix/return the item, the children playing together, and the target child making an assertive statement or asking a question why the child acted the way $\mathrm{s} /$ he did. For both Group Entry and
Provocation situations, negative/aggressive goals involve aggression by the target child or rejection or aggression by peer(s). Examples of inept goals include crying or pouting and telling an adult. Walking away from the situation or wanting nothing to happen are examples of nonsocial goals. Responses that are unrelated to the story or do not attempt to deal with the situation are coded as irrelevant goals.

Response Generation: For Group Entry situations, competent responses include waiting and hovering, requesting to join in the activity, making a statement about the activity (e.g., "That looks like fun"), asking the children a question, or bargaining with the peers. Inept responses include making a self-referent statement (e.g., "I went to Disney Land"), engaging in an alternate activity, seeking adult assistance, doing nothing, or leaving the situation. Any response involving threats, disruption of the activity, or verbal or physical aggression is coded as an aggressive response. For Provocation situations, competent responses include asking the child a question (e.g., "Why did you do that?"), making an assertive statement (e.g., "I don't think that was nice."), or sharing or being nice to the other child. Examples of inept responses include seeking adult assistance, blaming the other child, crying, or refusing to be the peer's friend anymore. Any response involving threats or verbal or physical aggression is coded as an aggressive response.

Enactment: To receive a score of 4, a child role plays with relevant content and has impeccable facial expression and tonal quality. A child who received a 3 on enactment role plays with relevant content and has both appropriate eye contact and tonal quality. Appropriate eye contact is defined as looking at the examiner for the majority of the enactment. To have appropriate tonal quality a child must speak in a clear and understandable manner in a tone that is loud enough for normal conversation. To receive a score of 2, a child uses relevant content, but lacks either appropriate eye contact or tonal quality. A child who receives a 1 role plays but uses inappropriate or irrelevant content, leaves out large sections of the content, or lacks both eye contact and tonal quality. Children who do not role play receive a score of 0 .

\section{References}

Bishop, S., Gahagan, S., \& Lord, C. (2007). Re-examining the core features of autism: A comparison of autism spectrum disorder and fetal alcohol spectrum disorder. Journal of Child Psychology and Psychiatry, and Allied Disciplines, 48(11), 1111-1121. doi:10.1111/j.1469-7610.2007.01782.x.

Bowers, L., Huisingh, R., \& LoGiudice, C. (2005). Test of problem solving 3 (TOPS 3: elementary): a test of reasoning in context. East Moline, IL: LinguiSystems, Inc.

Burks, V. S., Laird, R. D., Dodge, K. A., Pettit, G. S., \& Bates, J. E. (1999). Knowledge structures, social information processing, and 
children's aggressive behavior. Social Development, 8(2), 220236. doi:10.1111/1467-9507.00092.

Coggins, T. E., Friet, T., \& Morgan, T. (1998). Analyzing narrative productions in older school-age children and adolescents with fetal alcohol syndrome: An experimental tool for clinical applications. Clinical Linguistics \& Phonetics, 12(3), 221-236. doi:10.3109/02699209808985223.

Cohen, J. (1988). Statistical power analysis for the behavioral sciences (2nd ed.). Hillsdale, N.J.: Erlbaum.

Crick, N. R., \& Dodge, K. A. (1994). A review and reformulation of social information-processing mechanisms in children's social adjustment. Psychological Bulletin, 115(1), 74-101. doi:10.1037/ 0033-2909.115.1.74.

Dodge, K. A. (1980). Social cognition and children's aggressive behavior. Child Development, 51(1), 162-170. doi:10.2307/ 1129603.

Dodge, K. A., Laird, R., Lochman, J. E., Zelli, A., \& Group, Conduct Problems Prevention Research. (2002). Multidimensional latentconstruct analysis of children's social information processing patterns: Correlations with aggressive behavior problems. Psychological Assessment, 14(1), 60-73. doi:10.1037/10403590.14.1.60.

Dodge, K. A., Lansford, J. E., Burks, V. S., Bates, J. E., Pettit, G. S., Fontaine, R., et al. (2003). Peer rejection and social informationprocessing factors in the development of aggressive behavior problems in children. Child Development, 74(2), 374-393. doi:10.1111/1467-8624.7402004.

Gifford-Smith, M. E., \& Rabiner, D. L. (2004). Social information processing and children's social adjustment. In J. B. Kupersmidt \& K. A. Dodge (Eds.), Children's Peer Relations: From Development to Intervention, pp. 61-79. Washington, DC: American Psychological Association.

Hollingshead, A. B. (1975). Four factor index of social status. Unpublished working paper.

Hoyme, H. E., May, P. A., Kalberg, W. O., Kodituwakku, P., Gossage, J. P., Trujillo, P. M., et al. (2005). A practical clinical approach to diagnosis of fetal alcohol spectrum disorders: Clarification of the 1996 Institute of Medicine criteria. Pediatrics, 115, 39-47. doi:10.1542/peds.2005-0702.

Jones, K. L., \& Smith, D. W. (1973). Recognition of the fetal alcohol syndrome in early infancy. Lancet, 2(7836), 999-1001. doi:10.1016/S0140-6736(73) 91092-1.

Keil, V., \& Price, J. M. (2009). Social information-processing patterns of maltreated children in two social domains. Journal of Applied Developmental Psychology, 30, 43-52. doi:10.1016/j.appdev.2008.10.003.

Kodituwakku, P. W. (2007). Defining the behavioral phenotype in children with fetal alcohol spectrum disorders: A review. Neuroscience and Biobehavioral Reviews, 31(2), 192-201. doi:10.1016/j.neubiorev.2006.06.020.

Kupersmidt, J. B., \& DeRosier, M. E. (2004). How peer problems lead to negative outcomes: An integrative mediational model. In J. B. Kupersmidt \& K. A. Dodge (Eds.), Children's peer relations: from development to intervention, pp. 119-138. Washington, D. C.: American Psychological Association.

Mattson, S. N., Riley, E. P., Gramling, L. J., Delis, D. C., \& Jones, K. L. (1998). Neuropsychological comparison of alcohol-exposed children with or without physical features of fetal alcohol syndrome. Neuropsychology, 12(1), 146-153. doi:10.1037/ 0894-4105.12.1.146.

Maxwell, S. E., \& Delaney, H. D. (2004). Designing experiments and analyzing data (2nd ed.). Mahwah, N.J.: Erlbaum.

McGee, C. L., Fryer, S. L., Bjorkquist, O., Mattson, S. N., \& Riley, E. P. (2008). Social problem solving deficits in adolescents with prenatal exposure to alcohol. The American Journal of Drug and Alcohol Abuse, 34, 423-431. doi:10.1080/00952990802122630.

O'Connor, M. J., Frankel, F., Paley, B., Schonfeld, A. M., Carpenter, E., Laugeson, E. A., et al. (2006). A controlled social skills training for children with fetal alcohol spectrum disorders. Journal of Consulting and Clinical Psychology, 74(4), 639648. doi:10.1037/0022-006X.74.4.639.

Price, J. M., \& Landsverk, J. (1998). Social information-processing patterns as predictors of social adaptation and behavior problems among maltreated children in foster care. Child Abuse \& Neglect, 22(9), 845-858. doi:10.1016/S0145-2134(98) 00072-6.

Quiggle, N. L., Garber, J., Panak, W. F., \& Dodge, K. A. (1992). Social information processing in aggressive and depressed children. Child Development, 63(6), 1305-1320. doi:10.2307/1131557.

Redmond, S. M., \& Rice, M. L. (1998). The socioemotional behaviors of children with SLI: Social adaptation or social deviance? Journal of Speech, Language, and Hearing Research: JSLHR, $41,688-700$.

Rubin, K. H., \& Krasnor, L. R. (1986). Social-cognitive and social behavioral perspectives on problem solving. In M. Perlmutter (Ed.), Cognitive Perspectives on Children's Social and Behavioral Development (Vol. 18), pp. 1-68. Erlbaum: Hillsdale. NJ.

Sampson, P. D., Streissguth, A. P., Bookstein, F. L., Little, R. E., Clarren, S. K., Dehaene, P., et al. (1997). Incidence of fetal alcohol syndrome and prevalence of alcohol-related neurodevelopmental disorder. Teratology, 56(5), 317-326. doi:10.1002/ (SICI) 1096-9926(199711) 56:5 \&lt;317::AID-TERA5\&gt;3.0. $\mathrm{CO} ; 2-\mathrm{U}$.

Streissguth, A. P., Barr, H. M., Kogan, J., \& Bookstein, F. L. (1996). Final report: understanding the occurrence of secondary disabilities in clients with fetal alcohol syndrome (FAS) and fetal alcohol effects (FAE). Seattle, WA: University of Washington Publication Services.

Thomas, S. E., Kelly, S. J., Mattson, S. N., \& Riley, E. P. (1998). Comparison of social abilities of children with fetal alcohol syndrome to those of children with similar IQ scores and normal controls. Alcoholism, Clinical and Experimental Research, 22(2), 528-533.

Whaley, S. E., O'Connor, M. J., \& Gunderson, B. (2001). Comparison of the adaptive functioning of children prenatally exposed to alcohol to a nonexposed clinical sample. Alcoholism, Clinical and Experimental Research, 25(7), 1018-1024. doi:10.1111/ j.1530-0277.2001.tb02311.x. 